\title{
From chronic pruritus to neuroendocrine tumor: A case report
}

\author{
ALEXANDR CEASOVSCHIH ${ }^{1,2}$, GIORGIANA VOLOC ${ }^{2}$, VICTORIȚA ȘORODOC ${ }^{1,2}$, DAN VÂȚA $\breve{A}^{1,3}$, \\ CRISTIAN-DUMITRU LUPAȘCU ${ }^{1,4}$, CRISTINA PREDA $^{1,5}$, CĂTĂLINA LIONTE $^{1,2}$, ALEXANDRA STOICA $^{1,2}$, \\ OANA SIRBU ${ }^{1,2}$, ELENA-DANIELA GRIGORESCU ${ }^{1,6}$, RALUCA ECATERINA HALIGA $^{1,2}$, \\ ADORATA ELENA COMAN ${ }^{1,2}$, CRISTINA BOLOGA ${ }^{1,2}$, LUMINIȚA GINA VÂȚ $\breve{~}^{1,2}$, OVIDIU RUSALIM PETRIȘ ${ }^{1,2}$, \\ GABRIELA PUHA $^{1,2}$, GABRIELA DUMITRESCU ${ }^{1,2}$, MIHAI CONSTANTIN $^{1,2}$ and LAURENȚIU ȘORODOC ${ }^{1,2}$ \\ ${ }^{1}$ Department of Internal Medicine, Faculty of Medicine, 'Grigore T. Popa' University of Medicine and Pharmacy, \\ 700115 Iasi; ${ }^{2}$ Department of Internal Medicine, 'Sf. Spiridon' Clinical Emergency Hospital, 700111 Iasi; \\ Departments of ${ }^{3}$ Dermatology, ${ }^{4}$ Surgery, ${ }^{5}$ Endocrinology, and ${ }^{6}$ Diabetology, Faculty of Medicine, \\ ‘Grigore T. Popa’ University of Medicine and Pharmacy, 700115 Iasi, Romania
}

Received October 15, 2021; Accepted November 15, 2021

DOI: $10.3892 /$ etm.2022.11112

\begin{abstract}
Chronic pruritus is a major and distressing symptom of many diseases of dermatological, neurological, psychogenic or systemic origin. This chronic itch could be a presenting sign of malignancy; therefore, paraneoplastic pruritus has also been associated with neuroendocrine tumors (NETs). This article focuses on a patient presenting with chronic pruritus for the past 12 months and who received numerous treatment schemes with very poor clinical improvement, that presented in the hospital for worsening of the chronic pruritus associated with skin rash and significant weight loss (approximately $6 \mathrm{~kg}$ over a 2-month period). The laboratory tests showed iron deficiency anemia, eosinophilia and negative tumor markers. In order to investigate the hypoanabolic and anemic syndromes, upper gastrointestinal endoscopy and colonoscopy, which showed no lesions or tumors, were employed. Skin biopsy was performed and antihistaminic and local steroid treatment was initiated. The patient's status worsened within a week and the patient was started on systemic steroid treatment with poor results. Computer tomography was performed to identify any tumor(s) located either in the pelvis or abdomen. A lesion was found in the terminal ileum, identified as a hypervascularized associating bulky lymphadenopathy. The patient was transferred to the surgical ward where right hemicolectomy with manual ileotransverse anastomosis L-L was performed. The histopathological result confirmed NET G2. The patient clinically improved, the skin lesions resolved and the itchiness
\end{abstract}

Correspondence to: Dr Alexandr Ceasovschih or Dr Victorița Șorodoc, Department of Internal Medicine, Faculty of Medicine, 'Grigore T. Popa' University of Medicine and Pharmacy, 16 University Street, 700115 Iasi, Romania

E-mail: alexandr.ceasovschih@yahoo.com

E-mail: vivisorodoc@yahoo.com

Key words: pruritus, itch, urticaria, paraneoplastic, neuroendocrine tumor(s), malignancy, tumor markers disappeared. The general status improved significantly. NET G2 diagnosing was possible due to the atypic paraneoplastic sign: chronic pruritus. This case study highlights the association between itch and malignancy and presents an atypical way of NET presentation when all tumor markers remain negative.

\section{Case report}

A 77-year-old male non-smoker, without significant personal pathological history, presented to the Second Department of Internal Medicine of the 'Sf. Spiridon' Emergency Clinical Hospital, Iasi, Romania, complaining of chronic pruritus associated with skin rash for 12 months and significant weight loss; approximately $6 \mathrm{~kg}$ over a 2 month-period. The patient reported that the itch was not alleviated by any medication prescribed by the allergy specialist; several courses of antihistamine, antimycotic and antibiotic schemes resulted in worsening symptoms. The patient attributed the unintentional weight loss to a special diet which he had been following for 2 months in order to manage his pruritus.

On initial physical examination, the patient's good general status was observed; he was perfectly conscious and had no fever. His weight was $59 \mathrm{~kg}$ (versus current weight of $65 \mathrm{~kg}$ ), with an ideal body weight (IDW) equal to $71 \mathrm{~kg}$ and body mass index (BMI) equal to $18.41 \mathrm{~kg} / \mathrm{m}^{2}$. The patient experienced a slight loss of subcutaneous fat and muscle mass: apparent ribs, with a less pronounced, slight depression between them and with the clavicle bone region visible.

The skin examination revealed a few erythematous papules which were confluent in plaques and vaguely circumscribed; the lesions were itchy showing scaly skin with hematic crusts (Fig. 1).

The external chest was normal without lifts, heaves or thrills. The point of maximal impulse was not visible and was palpated in the 5th intercostal space at the midclavicular line. The heart rate and rhythm were normal, i.e., no murmurs, gallops, or rubs were auscultated. On pulmonary examination he presented no signs of lung fluid; the pulmonary murmur was normal. The abdominal examination revealed no significant 
abnormalities and normal bowel movement. The urogenital examination showed no dysuria, no nocturia, no change in frequency, no dribbling, and no signs of incontinence. Testicular examination showed no pain and no testicular abnormality.

The blood work performed showed a hemoglobin of $11.1 \mathrm{~g} / \mathrm{dl}$ confirming iron deficiency anemia, eosinophilia and negative tumor markers (carbohydrate antigen 19-9, $\alpha$ fetoprotein, carcinoembryonic antigen, cyfra 21-1). The abdominal ultrasound showed bilateral kidney stones. The chest X-ray revealed no pulmonary formations. Due to the fact that the patient had hypoanabolic syndrome and anemia, investigations continued with upper GI endoscopy which revealed gastroesophageal reflux (Fig. 2). Colonoscopy showed enlarged haustral folds (Fig. 3) and a few diverticular filling defects. There were no signs of tumors, inflammation or bleeding.

The patient was consulted in the Dermatology Clinic and the following diagnostic suspicions were raised: chronic eczema, herpetiform dermatitis, and chronic prurigo. Skin biopsy was performed and treatment was started. The patient received $4 \mathrm{mg} / \mathrm{day}$ of chlorphenamine and hydrocortison cream was applied 3 times per day.

The skin biopsy showed hyperkeratosis with ortho- and parakeratosis, hypergranulosis and inflammatory infiltrations, mainly limphoplasmocitary with perivascular distribution and few eosinophils and mast cells (Fig. 4).

The patient's status declined within a week, leading to systemic steroid being added to the medication. Considering that the patient's progress was unfavorable in the subsequent days, and the small intestine was not investigated, an abdominal and pelvic computed tomography (CT) scan was performed showing a tumoral mass located in the small bowel on the terminal ileum (Fig. 5).

The patient was transferred to the 2nd Surgery Department of the 'Sf. Spiridon' Emergency Clinical Hospital, Iasi, Romania, where right hemicolectomy with ileotransverse anastomosis was performed. The biopsy examination from the resected tumor fragment was classified according to the World Health Organization Classification and Suggested Grading of Neuroendocrine Neoplasms of the Digestive System classification-NET G2 (Fig. 6) (1). Microscopically, the tumor cells had round or oval nuclei with 'salt and pepper' chromatin and eosinophilic granular cytoplasm. The tumor nests were arranged in trabecular, insular, or sheet-like patterns. Glandular-like structures or palisading of the peripheral cell layers were occasionally evident (1).

The patient gradually improved clinically after the surgery and his prognosis was favorable on discharge. The skin lesions healed within two weeks. The hemoglobin levels improved and were normal shortly after the surgical intervention. Following surgery, the patient received adjuvant chemotherapy for 6 months with cisplatin and etoposide. The patient reported side effects which were managed using symptom release medication. At the follow-up appointments, the patient status remained favorable and the blood works were within normal range.

\section{Discussion}

One of the symptoms that can decrease the life of quality is pruritus (synonym: itch). A major impact on the quality of life can occur owing to different sensations perceived on the skin. Pruritus is an unpleasant sensory perception that gives an intense sensation of scratching $(2,3)$. Itch can occur using hematogenic or neurogenic mediators via multiple pathways, mainly located in the central nervous system. Pruritus may have a different origin being determined in the central nervous system via haematogenic or neurogenic mediators. In recent years, particular interest has been shown in the pathogenesis of pruritus, as well as in the nerves, receptors and mediators involved, leading to a better understanding thereof (4). Chronic pruritus is an itchy sensation that lasts for more than 6 weeks (5). It has been associated with neoplastic diseases and especially with lymphoproliferative diseases. Paraneoplastic itching has the following characteristics: i) it can appear even before the neoplastic evidence; ii) it is not caused by an invasive mass and iii) after the neoplasm is remitted the itching vanishes (6).

Malignancies can be accompanied by paraneoplastic symptoms or syndromes, which may be caused by an indirect effect of the malignancy and can represent the first sign of cancer (7). Pruritus appears as a sign at the beginning of the illness and is accentuated, particularly at night. The intensity of itching can reach a peak; thus, the patients with malignant tumors can associate real skin problems including excoriations, hyperpigmentation, lichenification or prurigo nodules. Paraneoplastic pruritus is most often associated with Hodgkin's lymphoma (the reported prevalence is 30\%) (8), and other lymphomas, including leukemia and polycythemia vera (9); however, studies show that neuroendocrine tumors (NETs) associate itch as well.

NETs are defined as heterogeneous tumors that originate in the cells with secretory function of the neuroendocrine system (10). In recent decades, in the US and around the world, the number of patients with NETs has increased considerably (11). In adults, NETs have an average diagnosis of 66 years of age with a higher incidence for women. In children, the incidence is approximately 0.995 cases per 100.000 in patients under 20 years of age. Non-specific symptoms of NETs lead to a low degree of diagnosis (12). Small bowel (SB)-NETs occur more often during the sixth and seventh decade of life. In 1867, Langhans was the first to describe a neuroendocrine tumor in the small intestine, a polypoid tumor in the small bowel identified at an autopsy. Research continued and in 1988, Lubarsch reported two cases of patients in whom numerous small tumors of the ileum were identified (13). The description of the first carcinoid syndrome in a patient who experienced diarrhea and dyspnea aggravated by food was made by Ransom, who at an autopsy identified that the patient had diffuse hepatic metastases and a distal ileal mass (14). However, most patients with NETs already have metastases at the time of diagnosis (15). Secondary metastatic lesions were found in approximately $21 \%$ of population-based studies during diagnosis confirmation (11). On the other hand, percentages as high as 56-69\% have been reported in retrospective chart reviews (16).

SB-NETs have a slow growth and cause metastases in the lymph nodes and the liver. The mortality caused by this tumor is in $80 \%$ of cases due to liver failure and in $16 \%$ due to bowel obstruction. The small size of SB-NETs that does not express a specific symptomatology determines a late diagnosis in the case of most patients. The majority of SB-NETs are identified at an advanced stage when the tumors cause symptoms specific to local complication. Enterochromaffin (EC) cells 

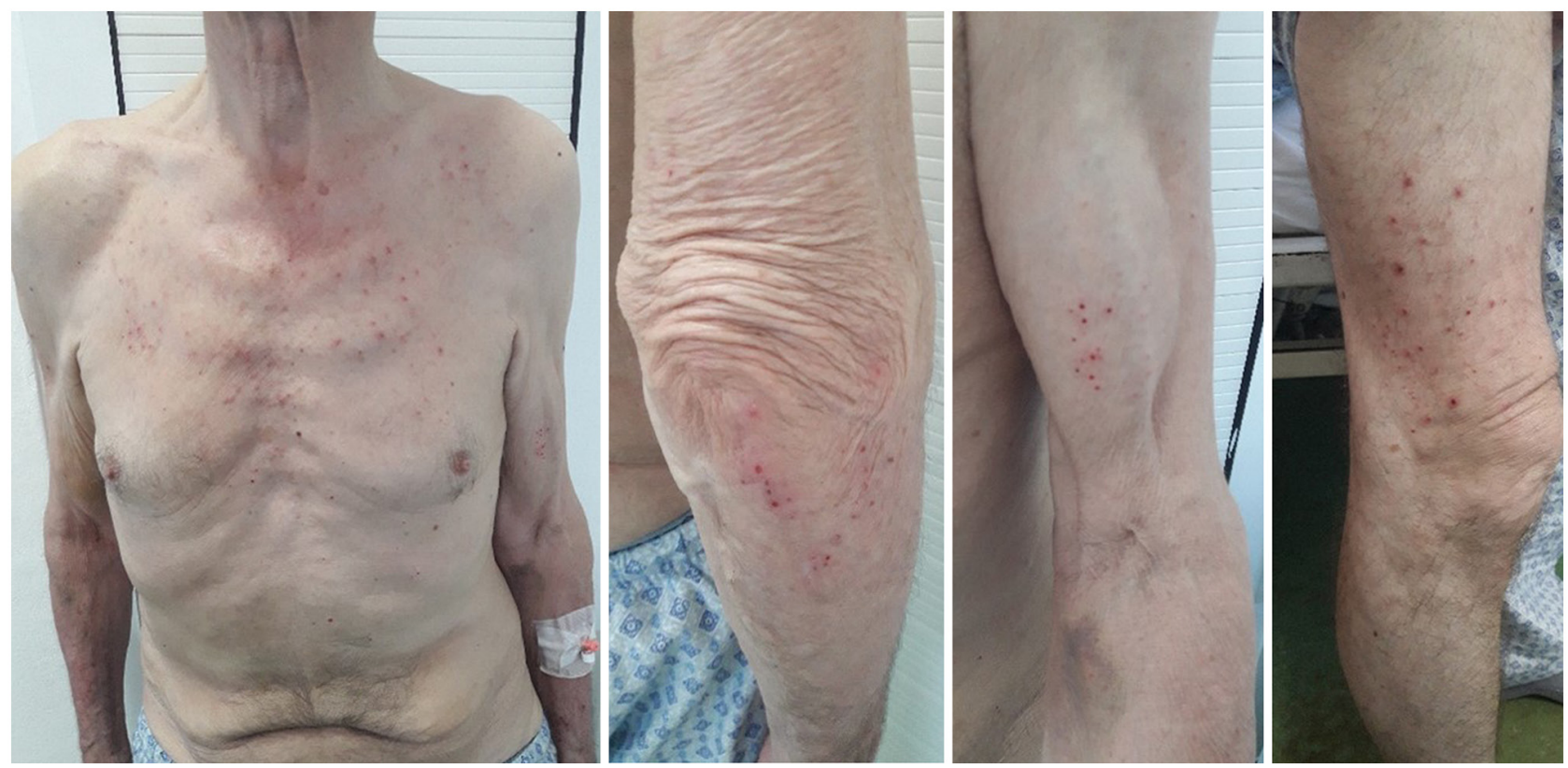

Figure 1. Erythematous papules, lesions with hematic crust located on the upper chest, lower and upper extremities.
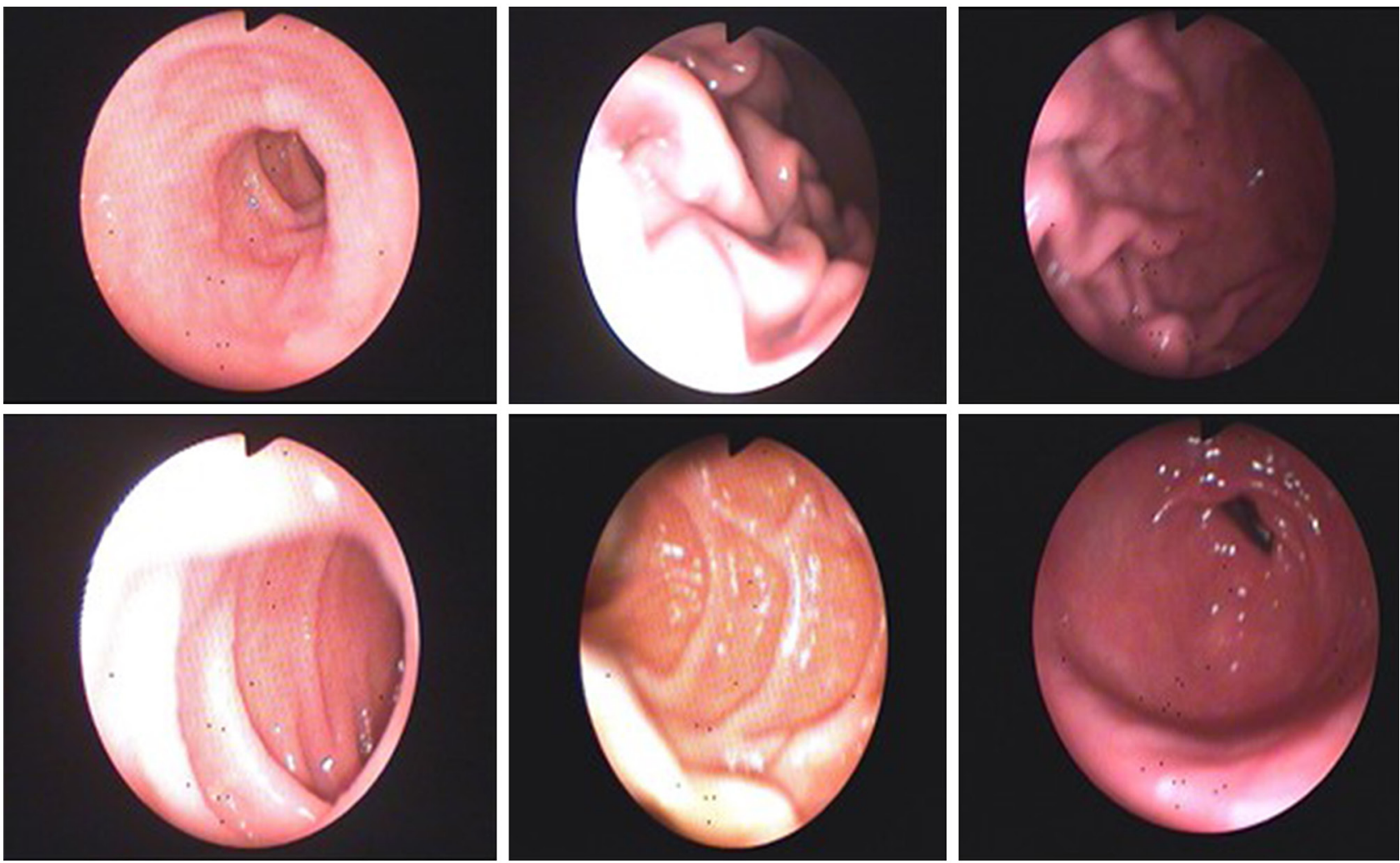

Figure 2. Upper GI endoscopy. Gastroesophageal reflux changes are evident following the upper GI endoscopy GI, gastrointestinal.

are responsible for 5-hydroxytryptamine (5-HT) secretion and are most often involved in NETs in the small intestine. 5-HT stored in EC cells is released under the action of various stimuli: mechanical stimulation, and nutrients and chemical stimulators, such as acetylcholine. Hypersecretion of serotonin from the small intestine NETs causes numerous symptoms, such as fatigue, diarrhea, flushes, bronchoconstriction, and feeling of squeezing or pressure in the chest. These symptoms summarize the carcinoid syndrome that is most common in advanced metastatic stages. The characteristic flush of NETs can lead to tumor localization. Cyanotic flushing associated with a burning sensation lasting no more than $1 \mathrm{~min}$ is associated with midgut tumors. Tumors with anterior location cause generalized pruritus (17).

CT is the imaging scan that can guide the diagnosis of NETs in the small intestine (18). CT detection sensitivity for NETs in 

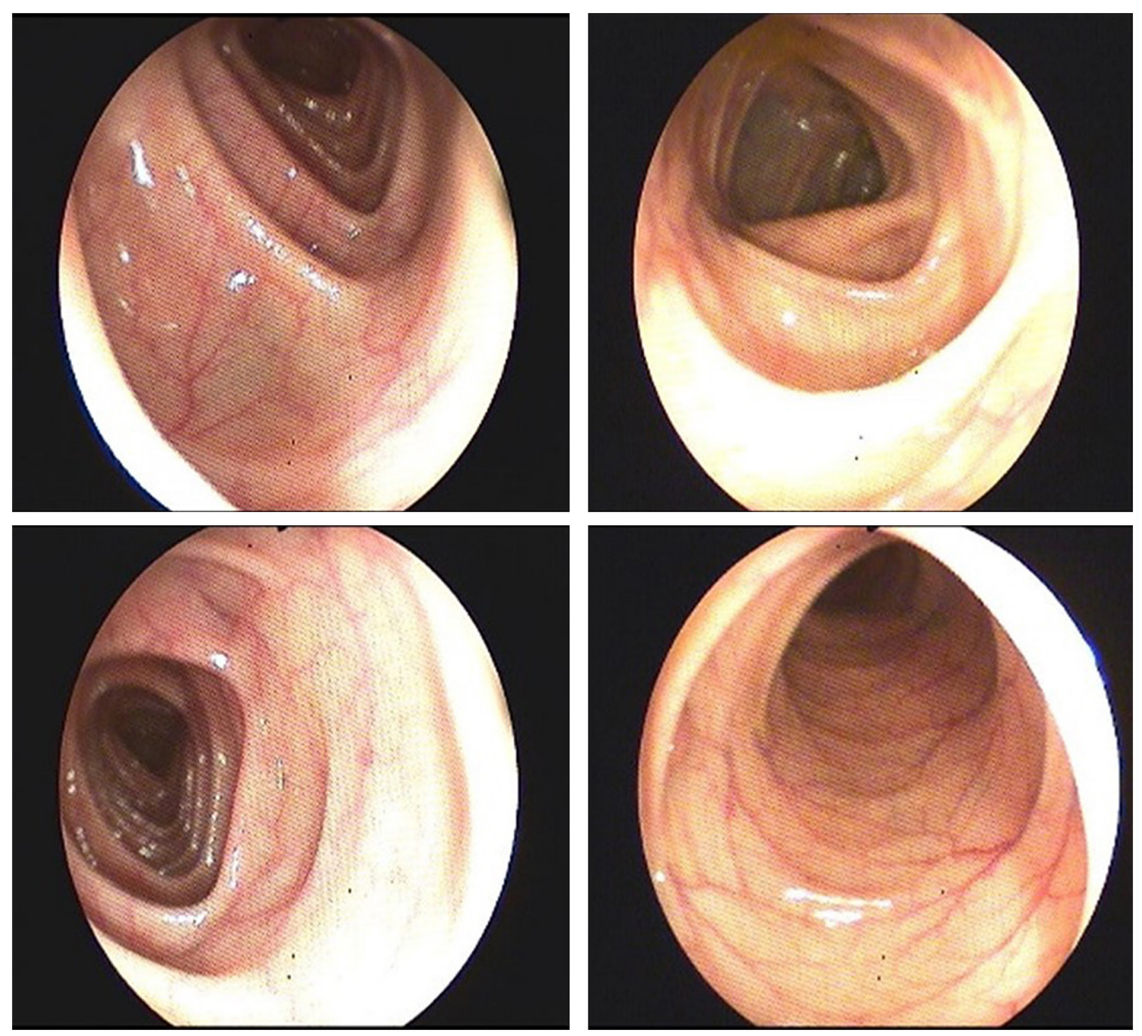

Figure 3. Colonoscopy. Enlarged haustral folds are evident.

A

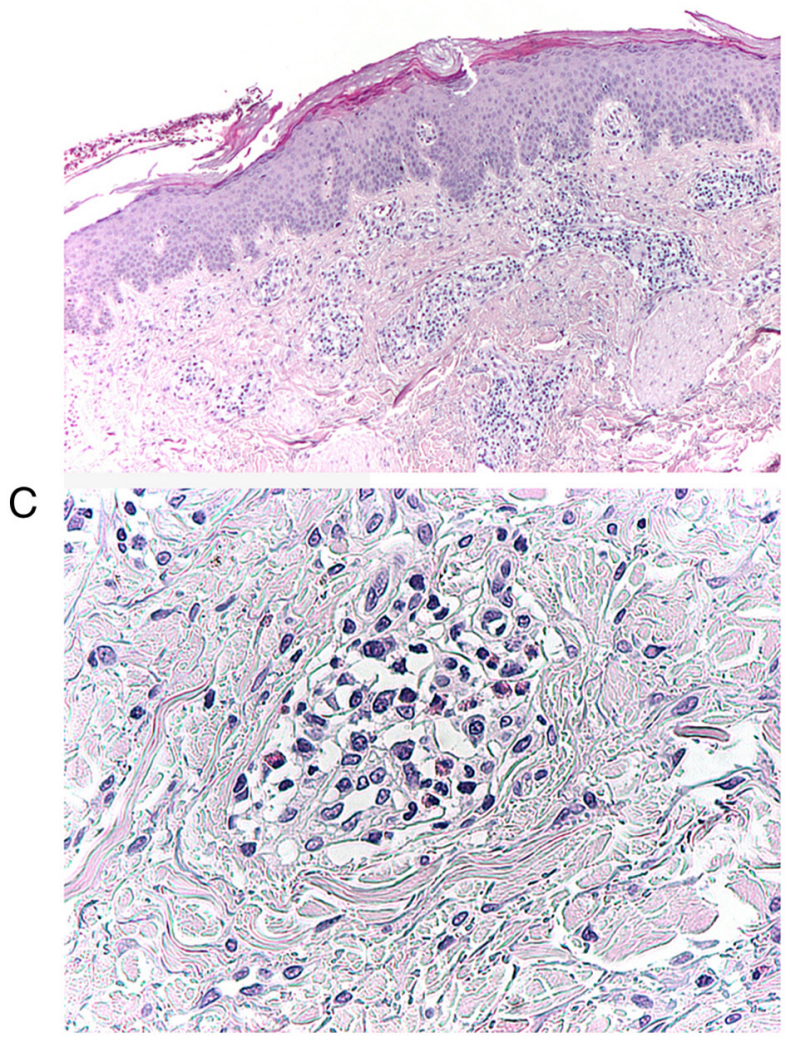

$\mathrm{B}$
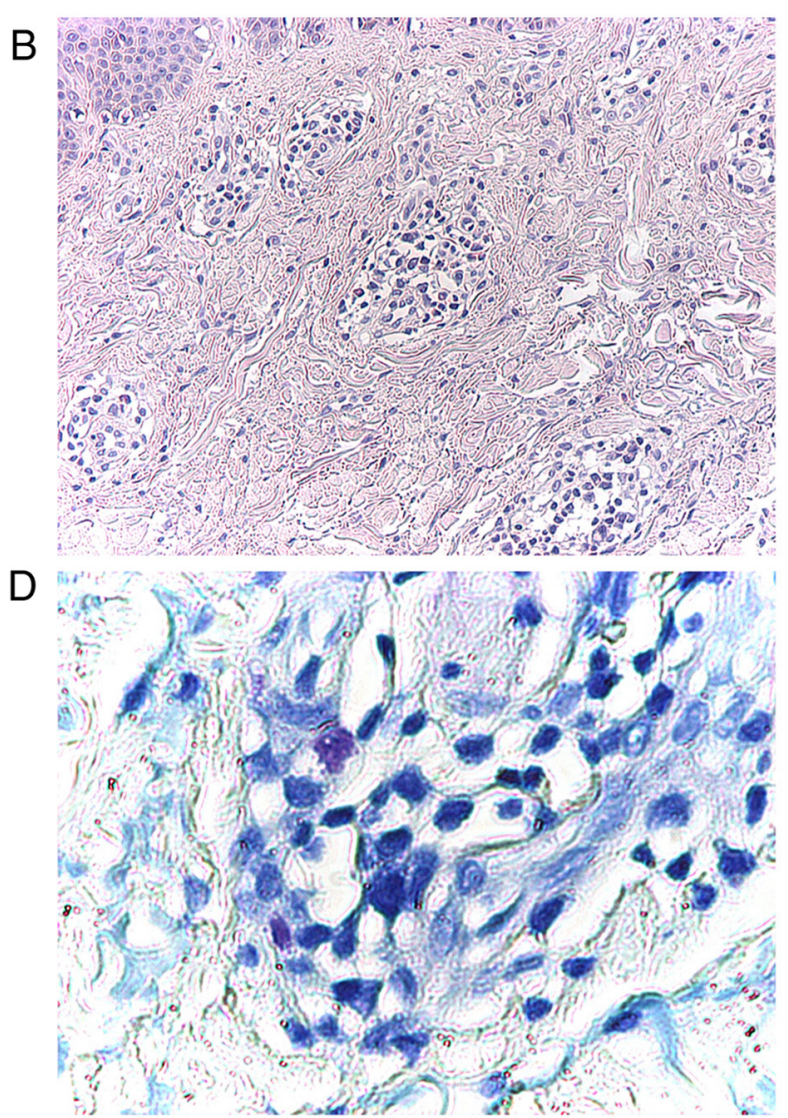

Figure 4. Skin biopsy results. (A) Hyperkeratosis with orthokeratosis described as thickening of the stratum corneum with parakeratosis seen as flattened keratinocyte nuclei within the stratum corneum and focal hypergranulosis observed as an increase in the intracellular granules of keratinocytes within the granular layer. H\&E; magnification, x4. (B) Inflammatory cell infiltration around the blood vessels with few eosinophils and mast cell infiltrate (perivascular infiltration). H\&E; magnification, x10. (C) Eosinophills. H\&E; magnification, x20. (D) Few mast cells with metachromatic cytoplasm (presence of cytoplasmic granules characterizes mast cells). AT; magnification, $\mathrm{x} 40$. 

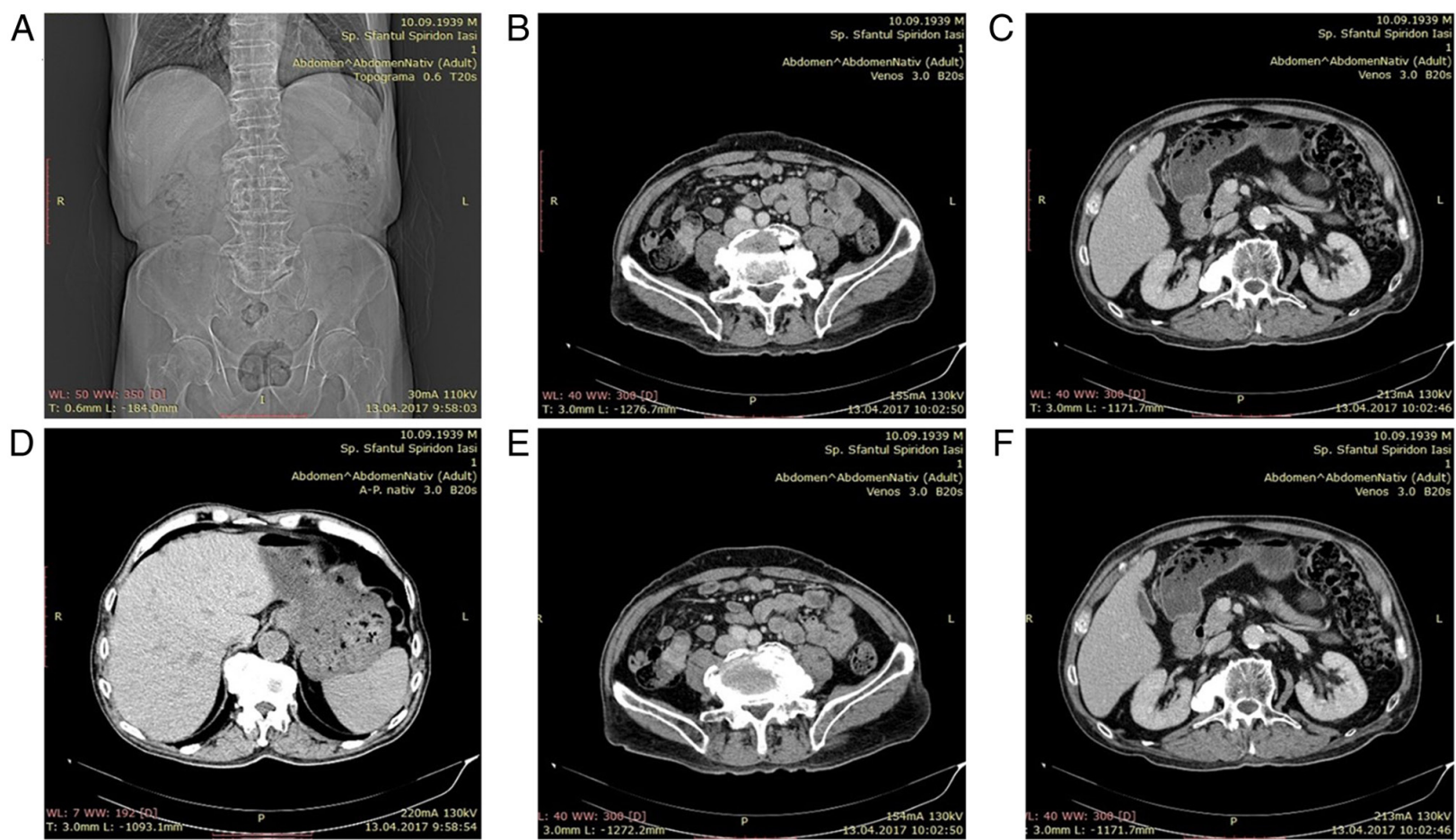

Figure 5. (A-F) Abdominal CT scan showing a mass on the terminal ileum of the small bowel.

A

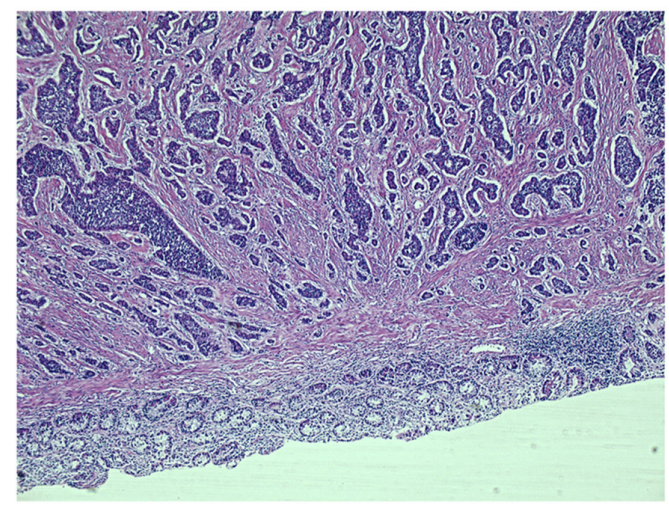

C

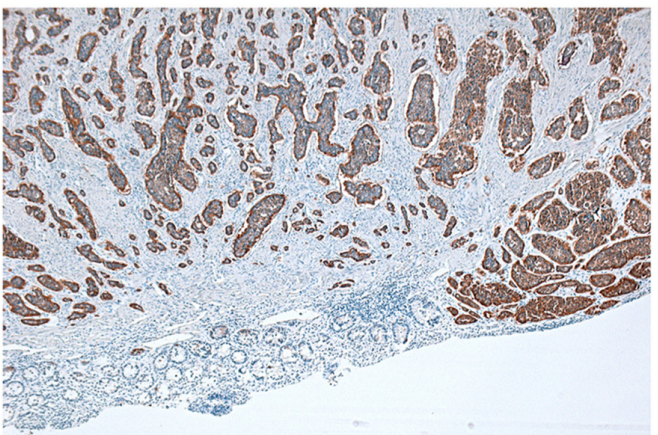

B
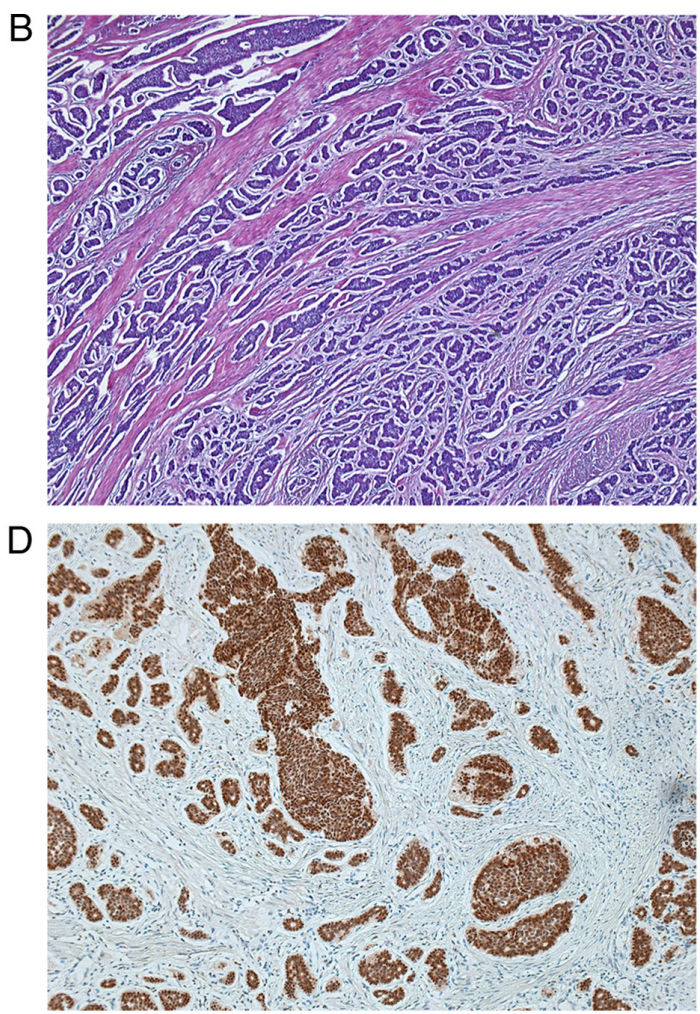

Figure 6. Histopathological images of NET G2 on anatomical specimen. (A and B) H\&E; magnification, x5 and x10, respectively. (C) Synaptophysin; magnification, x5. (D) CDX2; magnification, x10).

the small bowel is from 7 to $38 \%$. The sensitivity can reach to $82 \%$ if the presence of mesenteric lymphadenopathy/fibrosis is interpreted (19).

Circulating biomarker determination is considered useful to aid in diagnosis; however, it is not a mandatory determination for establishing a NETs diagnosis. Attempts have been made to measure some biomarkers, such as chromogranin A, pancreastatin, neurokinin A, neuron-specific enolase, pre-progastrin, pancreatic polypeptide, serotonin, and urinary or plasma 5-hydroxyindoleacetic acid; however, these biomarkers cannot 
provide data on tumor location. The differentiating usefulness between functional and non-functional tumors has been described by experts especially for patients with non-specific symptoms in which a carcinoid syndrome, induced by neuroendocrine tumors, is suspected. No consensus has been reached on the value of plasma biomarkers and tumor grade. Measurements of circulating biomarkers cannot differentiate low-level malignancy from high-grade disease (20).

Patients with NETs have a range of therapeutic options that must be adapted to the stage and disease evolution degree, such as somatostatin receptor agonist blockade, targeted radionuclides, immunotherapy (interferon), cytotoxic chemotherapy, rationally designed targeted drugs, external radiation, interventional radiological approaches, and surgery (either for cure or palliative debulking) (21,22).

In conclusion, diagnosing NET G2 was possible due to the atypic paraneoplastic sign: chronic pruritus. While there are not many studies suggesting a clear bond between neoplasia and pruritus, it is worth discussing and researching this vast interdisciplinary domain. This case study highlights the association between itch and malignancy, and presents an atypical way of NETs presentation while tumor markers remained negative and unreliable.

\section{Acknowledgements}

The authors thank Dr Karina Bulavschi from the Regional Institute of Oncology, Iasi, Romania, for support in collecting patient data and Iulian Bârsan for technical support.

\section{Funding}

No funding was received.

\section{Availability of data and materials}

The datasets used and/or analyzed during the current study are available from the corresponding author on reasonable request.

\section{Authors' contributions}

LS, AC, VS, AS, DV, CP, EDG carried out the patient investigation and ORP, REH, AEC, CB, OS data curation and interpretation. CDL performed the surgical intervention. GV, CL, LGV carried out the writing and original draft preparation and revised it for important intellectual content. GV, GP, GD, MC performed the literature data review and LS, VS, AC finally reviewed the manuscript. AC and VȘ confirm the authenticity of all the raw data. All authors read and approved the final version of the manuscript. All authors contributed equally to this work.

\section{Ethics approval and consent to participate}

Not applicable.

\section{Patient consent for publication}

The patient signed informed written consent about hospitalization, diagnostic and treatment interventions, and future data publication.

\section{Competing interests}

The authors declare no competing interests.

\section{References}

1. Bosman FT, Carneiro F, Hruban RH and Theise ND: WHO Classification of Tumours of the Digestive System. 4th Edition, Volume 3, 2010.

2. Patel T and Yosipovitch G: Therapy of pruritus. Expert Opin Pharmacother 11: 1673-1682, 2010.

3. Pogatzki-Zahn E, Marziniak M, Schneider G, Luger TA and Stander S: Chronic pruritus: Targets, mechanisms and future therapies. Drug News Perspect 21: 541-551, 2008.

4. Grundmann S and Ständer S: Chronic pruritus: Clinics and treatment. Ann Dermatol 23: 1-11, 2011.

5. Rajagopalan M, Saraswat A, Godse K, Shankar DS, Kandhari S, Shenoi SD, Tahiliani S and Zawar VV: Diagnosis and management of chronic pruritus: An expert consensus review. Indian J Dermatol 62: 7-17, 2017.

6. Yosipovitch G: Chronic pruritus: A paraneoplastic sign. Dermatol Ther 23: 590-596, 2010.

7. Beigi M, Häberle M, Gschwendtner A, Baum U and Weisshaar E: Generalized chronic itch as a first sign of malignancy resembling paraneoplastic sensomotoric neuropathy. Acta Derm Venereol 98: 526-527, 2018

8. Villafranca JJ, Siles MG, Casanova M, Goitia BT and Dominguez AR: Paraneoplastic pruritus presenting with Hodgkin's lymphoma: A case report. J Med Case Rep 8: 300, 2014.

9. Alpsoy E: Paraneoplastic pruritus and paraneoplastic erythroderma. Türkderm 47 (Suppl 1): S65-S68, 2013.

10. Allan B, Davis J, Perez E, Lew J and Sola J: Malignant neuroendocrine tumors: Incidence and outcomes in pediatric patients. Eur J Pediatr Surg 23: 394-399, 2013.

11. Yao JC, Hassan M, Phan A, Dagohoy C, Leary C, Mares JM, Abdalla EK, Fleming JB, Vauthey JN, Rashid A and Evans DB: One hundred years after 'carcinoid': Epidemiology of and prognostic factors for neuroendocrine tumors in 35825 cases in the United States. J Clin Oncol 26: 3063-3072, 2008.

12. Khanna G, O'Dorisio SM, Menda Y, Kirby P, Kao S and Sato Y: Gastroenteropancreatic neuroendocrine tumors in children and young adults. Pediatr Radiol 38: 251-259, quiz 358-9, 2008

13. Scott AT and Howe JR: Management of small bowel neuroendocrine tumors. J Oncol Pract 14: 471-482, 2018.

14. Ransom WB: A case of primary carcinoma of the ileum. Lancet 136: 1020-1023, 1890.

15. Hallet J, Law CH, Cukier M, Saskin R, Liu N and Singh S: Exploring the rising incidence of neuroendocrine tumors: A population-based analysis of epidemiology, metastatic presentation, and outcomes. Cancer 121: 589-597, 2015.

16. Raphael MJ, Chan DL, Law C and Singh S: Principles of diagnosis and management of neuroendocrine tumours. CMAJ 189: E398-E404, 2017.

17. Forero Molina MA, Garcia E, Gonzalez-Devia D, Garcia-Duperly R and Vera A: A 17-year-old male with a small bowel neuroendocrine tumor: Flushing differential diagnosis. World Allergy Organ J 10: 30, 2017.

18. Maxwell JE and Howe JR: Imaging in neuroendocrine tumors: An update for the clinician. Int J Endocr Oncol 2: 159-168, 2015.

19. Keck KJ, Maxwell JE, Menda Y, Bellizzi A, Dillon J, O'Dorisio TM and Howe JR: Identification of primary tumors in patients presenting with metastatic gastroenteropancreatic neuroendocrine tumors. Surgery 161: 272-279, 2017.

20. Oberg K, Modlin IM, De Herder W, Pavel M, Klimstra D, Friling A, Metz DC, Heaney A, Kwekkeboom D, Strosberg J, et al: Consensus on biomarkers for neuroendocrine tumour disease. Lancet Oncol 16: e435-e446, 2015.

21. Pavel M: Translation of molecular pathways into clinical trials of neuroendocrine tumors. Neuroendocrinology 97: 99-112, 2013.

22. Levy AD and Sobin LH: From the archives of the AFIP: Gastrointestinal carcinoids: Imaging features with clinicopathologic comparison. Radiographics 27: 237-257, 2007.

This work is licensed under a Creative Commons Attribution-NonCommercial-NoDerivatives 4.0 International (CC BY-NC-ND 4.0) License. 